\title{
Instrument and Method for Measuring Ice Accretion in Mixed-Phase Cloud Conditions
}

\author{
$1^{\text {st }}$ Eero Molkoselkä \\ Optoelectronics and Measurement \\ Techniques unit \\ University of Oulu \\ Oulu, Finland \\ eero.molkoselka@oulu.fi
}

\author{
$2^{\text {nd }}$ Ville Kaikkonen \\ Unit of Measurement Technology \\ University of Oulu \\ Kajaani, Finland \\ ville.kaikkonen@oulu.fi
}

\author{
$3^{\text {rd }}$ Anssi Mäkynen \\ Optoelectronics and Measurement \\ Techniques unit \\ University of Oulu \\ Oulu, Finland \\ anssi.makynen@oulu.fi
}

\begin{abstract}
The ICEMET-sensor is a novel cloud droplet and particle imaging instrument which measures icing conditions by determining the number and sizes of the supercooled droplets in a known air volume. The sensor captures digital holograms from $0.5 \mathrm{~cm}^{3}$ sample volume with a maximum rate of $3.0 \mathrm{~cm}^{3} / \mathrm{s}$. This lensless imaging instrument uses a computational imaging method to reconstruct the shadow images of the objects in the measurement volume. The size, position and shape descriptors of the individual particles and droplets are calculated and saved into a database. This data can be used to separate between cloud droplets and other particles. The calculated features are used to determine the two essential parameters needed for ice accretion modeling according to the ISO 12494 icing standard: liquid water content (LWC) of the air and median volume diameter (MVD) of the droplets. The basic working principle of the sensor and the image processing method are described. The performance of the sensor was tested in a wind tunnel under mixed-phase icing conditions. The measured LWC and MVD values were used to model ice accretion using the ISO 12494 icing standard for rotating cylinders. The modeled ice accretions were compared with weighed ice masses obtained from the wind tunnel with the same sized cylinder. The results show that accurate droplet size measurement and separation between droplets and ice crystals are essential for estimating the ice accretion rate properly. Without filtering out the ice crystals, the calculated accretion rates were overestimated by $65.6 \%$ on average.
\end{abstract}

Keywords-Atmospheric measurements, cloud droplets, digital holography, ice accretion, ice crystals, icing, image analysis, $L W C, M V D$

\section{INTRODUCTION}

Atmospheric in-cloud icing of structures is a common challenge in cold regions. The phenomenon is caused by super-cooled cloud droplets that freeze on surfaces forming a layer of ice. When left unnoted, the ice load can build up for long periods eventually damaging the structure. Traditionally overhead power lines and towers have had the most issues because of in-cloud icing [1]. However, as wind power has gained more popularity, it has become clear that icing also has a negative impact on wind energy production. Even a small ice accretion on turbine blades is enough to alter its aerodynamic properties and result in the loss of power production. Higher loads may cause mass imbalance and high amplitude vibrations that could eventually damage the turbine [2]. This creates a demand to monitor and be able to predict in-cloud icing events in real time.

The ice accretion rate depends on multiple environmental

This work was supported in part by European Regional Development Fund (ERDF) under European Territorial Cooperation (ETC) program Interreg V A Nord through Grant NYPS 20202472 and in part by Regional Council of Lapland through Grant 126/00.01.05.24.02/2019. factors such as particle concentration of the air, wind speed and the surface properties of the icing structure [1]. In case of cloud droplets, liquid water content (LWC) is used to measure the mass of liquid water in a cubic meter of air. The icing rate also depends on the size of the particles because it affects the collision efficiency of the cloud droplets. In-cloud icing is mainly caused by droplets with a diameter of 10 to 30 microns [3], [4]. For droplets, the median volume diameter (MVD) can be used to approximate the size distribution by using a single number [5]. It refers to the point in droplet size distribution (DSD) where the cumulative water mass is half of the total water mass. Close to the ground level LWC and MVD are heavily dependent on the local geographical factors and, in situ measurements are required to obtain accurate data [1], [6].

Icing clouds are often the so called mixed-phase clouds which contain both liquid water droplets and ice crystals. Typically, the most of the icing models ignore the ice crystals because in conditions near ground level their concentration is not very high [6]. In addition, accurate shape information is required in order to correctly calculate their mass. Cloud droplets can be considered spherical which simplifies their volume approximation. Ice crystals on the other hand form different types of complex shapes [7]. With common icing measurement techniques, the shape retrieval is nearly impossible. Most of the methods commonly used cannot differentiate between ice crystals and droplets.

ISO 12494 icing standard describes a method for measuring ice accretion by using a rotating cylinder with a diameter of $30 \mathrm{~mm}$ and minimum length of $0.5 \mathrm{~m}$ [8]. The ice accretion is typically measured by weighing the cylinder either manually or automatically.

Several light scattering based instruments have been developed [9], [10]. The method works by focusing a laser beam on a small volume and measuring intensity changes caused by crossing particles. One drawback with the method is that typically the size of the sampling volume depends on the speed of the airflow. This means that accurate wind speed information is required. CDP-2 (Cloud Droplet Probe) is a light scattering instrument for airborne LWC and DSD measurements. The method has been found to have some uncertainty when it comes to droplet sizes and in mixedcloud conditions small ice crystals are often assumed to be droplets [10].

Digital holography based instruments have gained more popularity in the last decade [11]-[13]. The method allows the computational reconstruction of the shadow images of the objects inside the measurement volume. The individual particles can then be analyzed one at the time. The 3D 
position, shape and size information about the particles can be obtained. The method's advantage in particle imaging is that the measurement volume is constant and not wind speed dependent.

\section{MATERIALS AND METHODS}

\section{A. Estimating Ice Accretion}

The icing standard provides a framework for estimating the ice accretion of several types of structures such as masts, towers and cables [8]. According to the standard icing rate $d_{m} / d_{t}(\mathrm{~g} / \mathrm{s})$ can be estimated using (1).

$$
d_{m} / d_{t}=\eta_{1} \eta_{2} \eta_{3} \cdot w \cdot A \cdot v
$$

The parameter $w$ is the mass concentration of the particles, $A$ is the cross-sectional area of the object and $v$ is the velocity of the particles. $\eta_{1-3}$ are collision, sticking and accretion factors varying between 0 and 1 . In case of icing caused by cloud droplets the mass concentration is equal to the LWC. $\eta_{2}$ and $\eta_{3}$ are generally assumed to be 1 for droplets. $\eta_{1}$ represents the ratio of droplets that hit the icing object.

The standard also includes a model for approximating $\eta_{1}$ for cloud droplets and a cylinder shaped icing object. The parameters of the model are MVD, droplet velocity, cylinder diameter, air density, water density and the absolute viscosity of air. The densities and viscosity can be calculated when the temperature and pressure are known and the droplet velocity can be assumed to be equal to the wind speed.

\section{B. ICEMET System}

The ICEMET-sensor (Fig. 1) is a novel cloud droplet and particle imaging instrument designed for in-situ measurements in harsh icing conditions [14], [15]. It is capable of measuring objects captured in the hologram from 3-5 $\mu \mathrm{m}$ up to $1-2 \mathrm{~mm}$. The sensor has $500 \mathrm{~W}$ heating capacity to protect it from icing and it rotates freely to align itself correctly according to the wind direction.

The in-line holographic setup of the ICEMET-sensor is shown in Fig. 2 [16]. Unlike most other holographic instruments, the laser beam is not collimated but acts as a point source. This reduces the cost and amount of optical elements needed in the system. The image sensor used is a grayscale Sony IMX264 with resolution of $2448 \times 2048$ pixels and pixel size of $3.45 \mu \mathrm{m}$. The laser is a $660 \mathrm{~nm}$ wavelength diode from Ushio Opto Semiconductors Inc. The setup forms a cone shaped $0.5 \mathrm{~cm}^{3}$ measurement volume between the protective windows. The frame rate of the system is 6 images per second. The optical magnification $M$ changes linearly as a function of the object position along the z-axis and can be obtained with (2).

$$
M(z)=z_{t o t} /\left(z_{t o t}-z\right)
$$

The parameter $z$ is the object position and $z_{t o t}$ is the distance between the sensor and the laser source.

The reconstruction of digital holograms is typically a computationally expensive task because the images often have a high resolution and large depth of the field. However, with modern hardware and proper parallelization methods it is possible to achieve real time performance. Today, this can be done with an ordinary desktop computer using almost any available GPU (graphics processing unit).

We developed an image processing and particle analysis software called ICEMET-Server (Fig. 3) [17]. The software

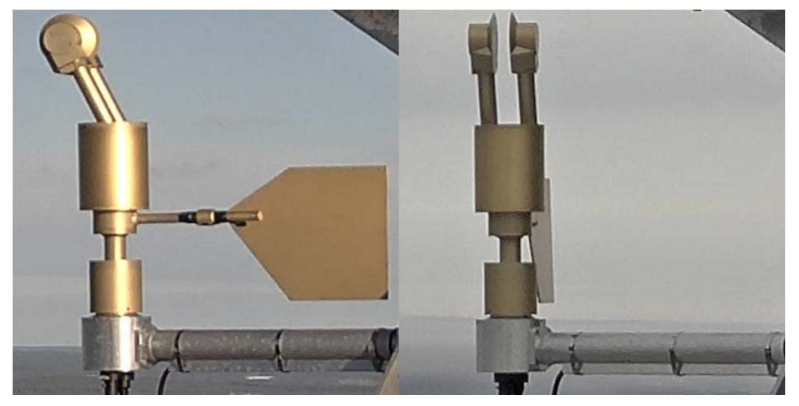

Fig. 1. The ICEMET-sensor on a wind turbine nacelle in February 2017.

Point source laser Image sensor

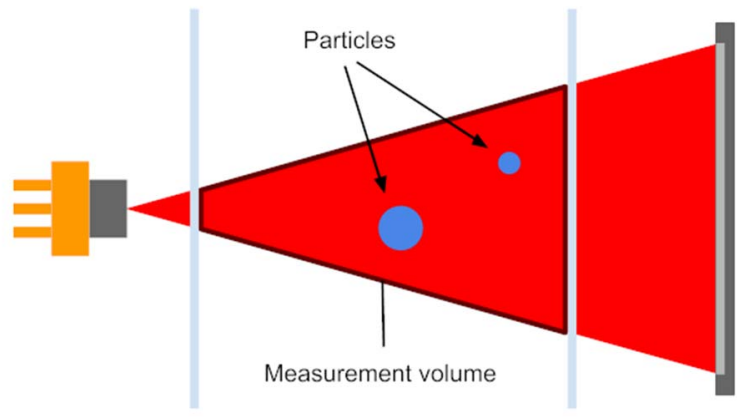

Protective window

Protective window

Fig. 2. The in-line holographic setup of the ICEMET-sensor. The distance between protective windows is $30 \mathrm{~mm}$.

is built with modern $\mathrm{C}++$ using OpenCV library and it is heavily multi-threaded and GPU accelerated [18]. Most of the algorithms developed are based on the ideas described by Fugal et al. [19].

\section{Image Processing}

The raw holographic images are preprocessed by center cropping them to resolution $2048 \times 2048$ pixels to remove the less illuminated image edges and to provide more uniform lighting across the hologram. Background subtraction is used to reduce the unwanted noise and further equalize the illumination. The image is divided by a background image and multiplied by its own mean pixel value. The background image is generated by taking the pixel-by-pixel median from a stack of seven consecutive holograms. The stack is updated every time a new hologram is captured which dynamically changes the background image over the time.

The preprocessed holographic images are reconstructed using angular spectrum propagation. The frequency domain filter $H$ is applied to reconstruct the hologram to distance $z$ (3).

$$
H(u, v, z)=\exp \left(-j k z \sqrt{1-(\lambda u)^{2}-(\lambda v)^{2}}\right)
$$

The variables $u$ and $v$ are the distances from the center of the image, $\lambda$ is the wavelength of the laser, $j$ the imaginary unit and $k$ the wavenumber [20]. The whole $30 \mathrm{~mm}$ sampling depth is reconstructed into a stack of 1500 images. Simultaneously, a new same sized image containing the minimum pixel values found in the whole stack for each pixel is created. The minimum image is formed by first selecting the first reconstructed hologram slice as the minimum image. Then the pixel values of the next 


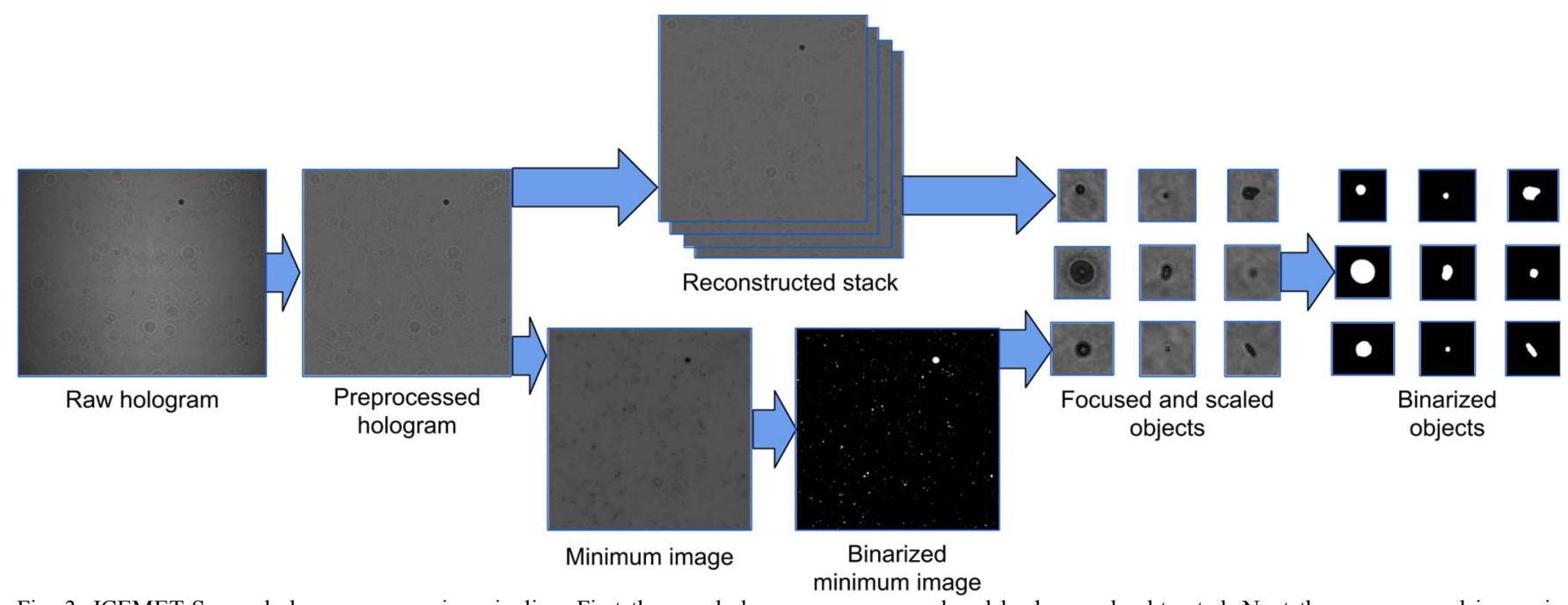

Fig. 3. ICEMET-Server hologram processing pipeline. First the raw holograms are cropped and background subtracted. Next the preprocessed image is reconstructed into a stack and minimum image. The individual particles are cropped using the binarized minimum image and focused. Finally the particles are scaled and binarized.

reconstructed hologram slice are compared with each other and the lower values are chosen as the new minimum image pixel values. The result will be an image containing all the lowest and thus darkest values for each pixel. Because the shadow images of the objects appear darker than the background, they will all show in the minimum image. The $2 \mathrm{D}$ coordinates and approximate sizes of the objects can be found by binarizing the minimum image and using basic contour detection. The information is used to crop the image into smaller rectangular segments.

The z-position of an object can be found by sliding the 2D segment through the reconstructed stack. A scoring function is used to find the best focus automatically. The process is often called autofocusing. An ideal scoring function has its global maximum at the z-position of the object and no local peaks. Many different functions have been proposed for autofocusing [19], [21], [22]. Through a testing process, two different scoring functions were chosen. For objects with width and height smaller than 5 pixels, the minimum intensity of the segment is used. The method chooses the z-position with the lowest pixel value. The minimum based autofocusing is fast and because most of the objects found will be small droplets, the processing times will be reduced significantly. However, the function won't be able to correctly score larger objects with more complex shapes. For them, the maximum standard deviation of standard deviation filtered (SDoSDF) segment is used. The method applies a $3 \times 3$ standard deviation filter on the segment at every z-position and calculates the standard deviations of the filtered segments [23]. This method was found by experimenting with different autofocus functions. Many methods gave good results when used with snow flakes and other complex shapes but SDoSDF performed the best for circular droplets. To further accelerate the focusing process, a ternary search based greedy search algorithm is used to find the best score [24]. The method reduces the execution time approximately $90 \%$ in comparison to iterating through the whole stack.

\section{Particle Analysis and Statistics}

The perimeter calculation function of OpenCV uses the pixel centroids [18]. This makes the comparison of particles with different sizes difficult as the perimeters of smaller particles will be underestimated more relatively. The focused particle segments are scaled approximately to 200 pixels using Lanczos resampling [25]. This way the segment sizes will be approximately the same throughout the analysis. For the smaller particles, the upscaling brings up their sub-pixel features that makes determining their shape more feasible. The scaled particles are binarized using a threshold $I_{t h}$ (4).

$$
I_{t h}=I_{b g}-0.35\left(I_{b g}-I_{\min }\right)
$$

$I_{b g}$ is the global background and $I_{\min }$ the local minimum of the segment. $I_{b g}$ is calculated by taking the median of the preprocessed hologram. The constant 0.35 has been chosen through a calibration process with NIST traceable microspheres. The equivalent diameter is calculated using the area of the object. The perimeter $p$ is also extracted and used to together with the equivalent diameter to obtain object's circularity factor $f_{c}(5)$. The circularity factor is the real perimeter of an object divided by the perimeter derived from its area $A$ when the object is assumed to be a circle.

$$
f_{c}=p /(2 \sqrt{\pi A})
$$

A lower value means that the object is more circular. The factor can be used to distinguish circular droplets from unwanted objects. All the droplet diameters are inserted into DSD bins.

Because the sampling volume and the number of frames per second are known, LWC at every measurement interval can be obtained by summing up the water mass in all droplets and dividing it by the total volume. MVD is calculated from the DSD.

\section{E. The Icing Wind Tunnel Measurements}

The measurements were done in VTT icing wind tunnel 2.0 located in Espoo, Finland, in October 2016 [26]. The ICEMET-sensor was placed side by side with a rotating 0.5 $\mathrm{m}$ long standard cylinder as seen in Fig. 4. [8]. The accreted ice was weighed and removed after every measurement.

All the measurements and conditions are presented in Table 1. The parameters were temperature, wind speed and targeted LWC. The targeted LWC values were set by the wind tunnel operators by measuring and adjusting the water flow. The measurements \#3a-b and \#5a-b were made in the same conditions. The measurement durations were between 10 and 24 minutes. 


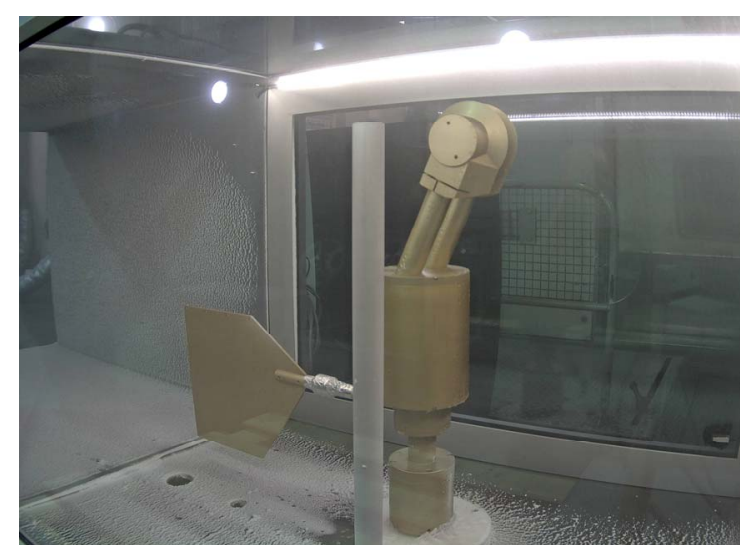

Fig. 4. The ICEMET-sensor and $0.5 \mathrm{~m}$ long standard cylinder in the VTT icing wind tunnel 2.0

TABLE I. MEASUREMENT CONDITIONS

\begin{tabular}{|l|l|l|l|}
\hline$\#$ & Temperature $\left({ }^{\circ} \mathbf{C}\right)$ & Wind speed $(\mathbf{m} / \mathbf{s})$ & LWC $\left(\mathbf{g} / \mathbf{m}^{3}\right)^{*}$ \\
\hline 1 & -5 & 7 & 0.2 \\
\hline 2 & -5 & 7 & 0.4 \\
\hline $3 a$ & -15 & 7 & 0.2 \\
\hline $3 b$ & -15 & 7 & 0.2 \\
\hline 4 & -15 & 10 & 0.2 \\
\hline $5 a$ & -15 & 7 & 0.4 \\
\hline $5 b$ & -15 & 7 & 0.4 \\
\hline 6 & -15 & 10 & 0.4 \\
\hline 7 & -15 & 20 & 0.4 \\
\hline
\end{tabular}

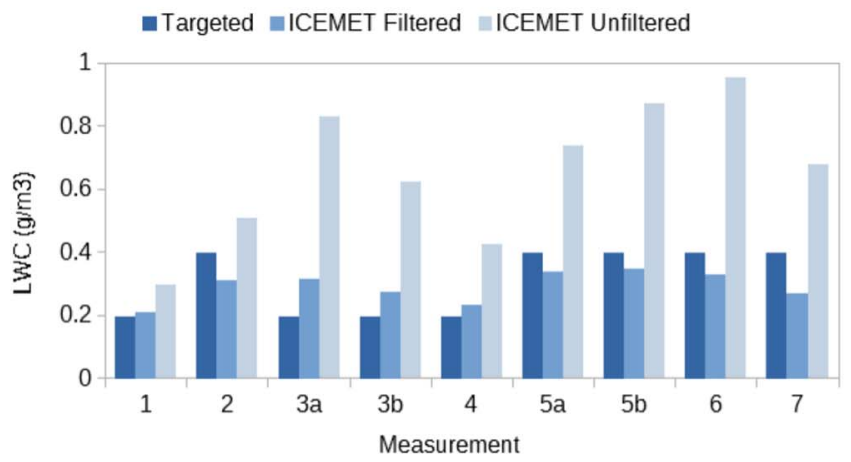

(a)

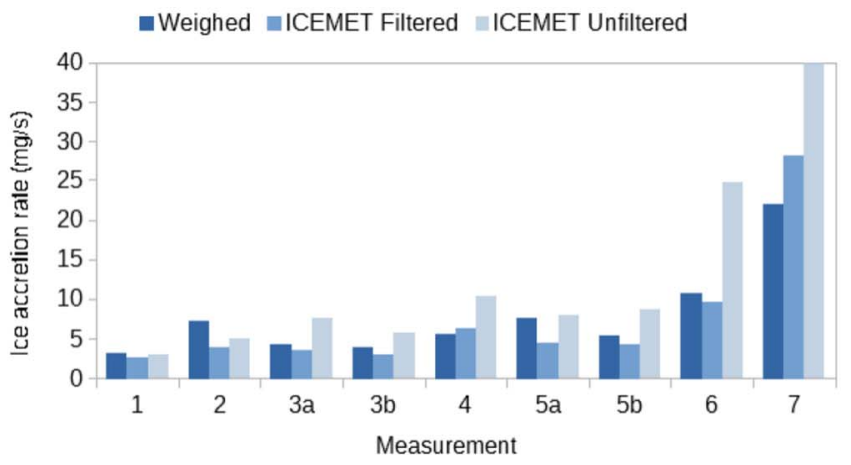

(c)

\section{RESULTS AND DISCUSSION}

The ICEMET-sensor's particle image data was analyzed with and without circularity factor filtering. With filtering enabled, all the particles with $f_{c}>1.07$ were pruned. The threshold was chosen manually by examining the particle images. The goal was to find a value where all the ice crystals would be removed but the number of droplets wouldn't be affected. The measurement results and the corresponding calculations are presented in Fig. 5. The targeted LWC values were compared with the values measured with the ICEMET-sensor. The corresponding MVD values measured with the ICEMET-sensor with both the filtered and unfiltered data are shown in Fig. 5. (b). The ice accretion rates were modeled with the icing standard using the wind speed data from the wind tunnel and the measured LWC and MVD values [8]. The values used in modeling based on the wind tunnel conditions: air density and the absolute viscosity $1.316 \mathrm{~kg} / \mathrm{m}^{3}$ and $1.69 \cdot 10^{-5} \mathrm{~kg} /(\mathrm{m} \cdot \mathrm{s})$ in $-5{ }^{\circ} \mathrm{C}$ and $1.367 \mathrm{~kg} / \mathrm{m}^{3}$ and $1.64 \cdot 10^{-5} \mathrm{~kg} /(\mathrm{m} \cdot \mathrm{s})$ in $-15^{\circ} \mathrm{C}$. The water density in both temperatures was $1000 \mathrm{~kg} / \mathrm{m}^{3}$. The icing rates $(\mathrm{mg} / \mathrm{s})$ from the rotating cylinder measurements were calculated from the accumulated total ice mass. The weighed ice load masses were between 3.7 and $20.0 \mathrm{~g}$.

In Fig. 5.(a) it can be seen that the calculated LWC values without the ice crystal filtering enabled were higher in every measurement and they were vastly overestimated in \#3-7. This was most likely caused by the large amount of ice crystals in $-15{ }^{\circ} \mathrm{C}$ temperature. When randomly shaped ice crystals are assumed to be spherical, their volume and mass will be largely overestimated. The LWC values where the ice crystals had been removed were closer to the targeted values. In theory, the measured LWC values should be smaller than the inputted water amount to the tunnel would predict if a part of the droplets turned into ice crystals.

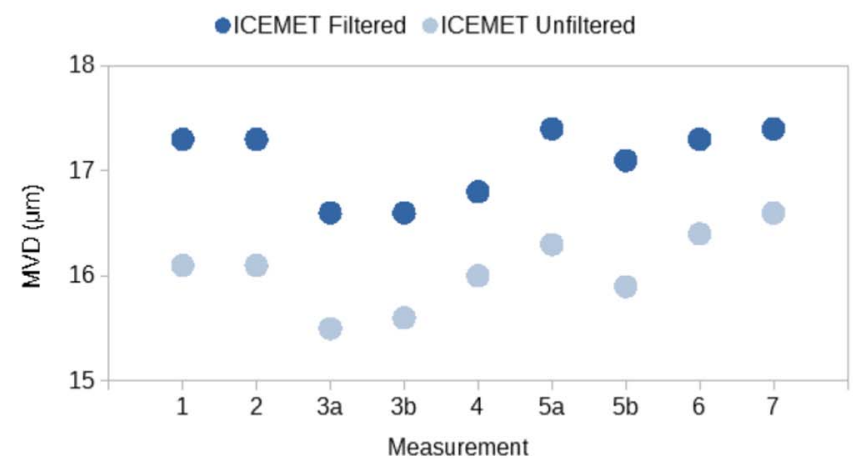

(b)

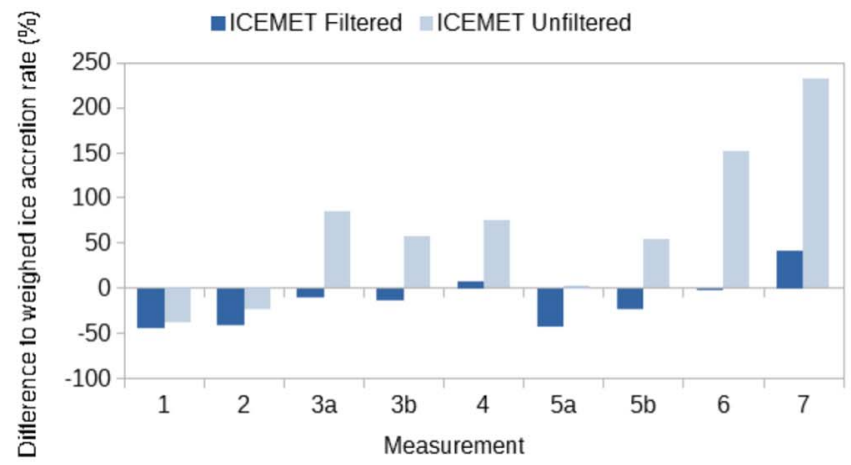

(d)

Fig. 5. Wind tunnel results. (a) LWC values estimated by VTT operators and values measured with the ICEMET-sensor with and without circularity filtering. (b) MVD values with and without filtering. (c) Weighed and calculated ice accretion rates. \#7 unfiltered. value is $66.4 \mathrm{mg} / \mathrm{s}$. (d) The percentage difference compared to the weighed accretion rate. 
The change in the wind speed and targeting the same LWC level requires adjusting of the waterflow in the tunnel, this is the most probable explanation for the slight differences in the measured LWC values when comparing results from \#3a-b with \#4 and \#5a-b with \#6 and \#7.

The MVD values are presented in Fig. 5. (b). The values were approximately $16.5-17.5 \mu \mathrm{m}$ for the filtered and 15.5$16.5 \mu \mathrm{m}$ for the unfiltered measurements. This indicates that the filtering reduced more the number of small particles than larger particles. The values were also within the size range of 10-30 $\mu \mathrm{m}$, the range that typically causes most of the icing [3], [4].

Ideally the filtering should remove all the ice crystals and leave only droplets for the LWC and MVD calculation. In Fig. 5. (c) it can be seen that for the unfiltered results, the estimated ice accretion rates were all much higher than those measured by the icing standard cylinder. This was expected as the LWC values were higher when the filtering was disabled. A higher wind speed increases the icing rate and thus it caused even a larger overestimation in measurements \#6 and \#7 between the filtered and unfiltered data.

The percentage difference compared with the weighed accretion is shown in Fig. 5. (d). On average, the unfiltered results were $65.6 \%$ higher than the icing rate of the standard cylinder. The worst result was made in the measurement \#7 where the estimated rate was $232.0 \%$ higher. The accretion rates with the ice crystal filtering enabled were much closer to the cylinder values and were $13.4 \%$ lower on average. This would suggest that the used $f_{c}$ threshold was slightly too strict. There is also the possibility that part of the ice accretion was caused by the ice crystals, which is neglected in the icing model.

In order to estimate icing in the mixed-phase clouds properly, a distinction between droplets and ice crystals must be made. Assuming that all the passing objects are droplets will result in skewed icing rates, especially with high wind speeds. With an imaging based droplet instruments, the filtering can be done with basic image analysis.

\section{CONCLUSION}

A novel digital holography based imager, the ICEMETsensor, was presented together with its image analysis software. The software has important functionality for separating cloud droplets from other particles such as ice crystals. The LWC and MVD values measured with the method can be used together with the ISO 12494 icing standard to realistically model ice accretion even in mixedphase cloud events. The data offers versatility to examination of mixed-phase icing. The distinct analysis of the cloud droplets and ice crystals can be potentially used to increase the accuracy of the icing models in future.

\section{ACKNOWLEDGMENT}

We would like to thank Mr. Timo Arstila, Mr. Timo Kananen and the involved VTT wind tunnel operators for performing the measurements.

\section{REFERENCES}

[1] L. Makkonen, "Models for the growth of rime, glaze, icicles and wet snow on structures," Philosophical Transactions of the Royal Society of London. Series A: Mathematical, Physical and Engineering Sciences, vol. 358, no. 1776, pp. 2913-2939, 2000.

[2] O. Parent and A. Ilinca, "Anti-icing and de-icing techniques for wind turbines: Critical review," Cold Regions Science and Technology, vol. 65, no. 1, pp. 88-96, 2011.
[3] L. Makkonen, "Analysis of Rotating Multicylinder Data in Measuring Cloud-Droplet Size and Liquid Water Content," Journal of Atmospheric and Oceanic Technology, vol. 9, no. 3, pp. 258-263, 1992.

[4] S. G. Cober, G. A. Isaac, and J. W. Strapp, "Characterizations of Aircraft Icing Environments that Include Supercooled Large Drops," Journal of Applied Meteorology, vol. 40, no. 11, pp. 1984-2002, 2001.

[5] K. J. Finstad, E. P. Lozowski, and L. Makkonen, "On the Median Volume Diameter Approximation for Droplet Collision Efficiency," Journal of the Atmospheric Sciences, vol. 45, no. 24, pp. 4008-4012, 1988.

[6] L. Makkonen, "Modeling of Ice Accretion on Wires," Journal of Climate and Applied Meteorology, vol. 23, no. 6, pp. 929-939, 1984.

[7] Korolev, A. V., et al. "Ice Particle Habits in Arctic Clouds." Geophysical Research Letters, vol. 26, no. 9, Jan. 1999, pp. 12991302.

[8] Atmospheric icing of structures, ISO standard 12494:2017, 2017.

[9] Baumgardner, D, et al. "The Cloud, Aerosol and Precipitation Spectrometer: a New Instrument for Cloud Investigations." Atmospheric Research, vol. 59-60, 2001, pp. 251-264.

[10] S. Lance, C. A. Brock, D. Rogers, and J. A. Gordon, "Water droplet calibration of the Cloud Droplet Probe (CDP) and in-flight performance in liquid, ice and mixed-phase clouds during ARCPAC," Atmospheric Measurement Techniques, vol. 3, no. 6, pp. 1683-1706, 2010.

[11] J. P. Fugal, R. A. Shaw, E. W. Saw, and A. V. Sergeyev, "Airborne digital holographic system for cloud particle measurements," Applied Optics, vol. 43, no. 32, p. 5987, Oct. 2004.

[12] J. Henneberger, J. P. Fugal, O. Stetzer, and U. Lohmann, "HOLIMO II: a digital holographic instrument for ground-based in situ observations of microphysical properties of mixed-phase clouds," Atmospheric Measurement Techniques, vol. 6, no. 11, pp. 2975-2987, Jun. 2013.

[13] S. Rydblom, B. Thörnberg, and E. Olsson, "Field study of lwc and mvd using the droplet imaging instrument," IEEE Transactions on Instrumentation and Measurement, 68(2), pp. 614-622, 2018.

[14] V. A. Kaikkonen, E. O. Molkoselkä, and A. J. Mäkynen. (Jun. 2019) ICEMET - a sensor for in-cloud icing condition monitoring. Presented at IWAIS 2019. [Online]. Available: https://iwais2019.is/images/Papers/081_Kaikkonen_Ville.pdf

[15] V. A. Kaikkonen, E. O. Molkoselkä, and A. J. Mäkynen, "ICEMET a sensor for in-cloud icing condition monitoring," in Proc. The 13th Japan-Finland Joint Symp. on Optics in Engineering, Aug. 2019, pp. 9-10.

[16] D. Gabor, "A New Microscopic Principle," Nature, vol. 161, no. 4098, pp. 777-778, 1948.

[17] ICEMET-Server. (2019). [Online]. Available: https://github.com/molkoback/icemet-server/

[18] OpenCV. (2019). [Online]. Available: https://opencv.org/

[19] J. P. Fugal, Schulz T.J. and R. A. Shaw, "Practical methods for automated reconstruction and characterization of particles in digital in-line holograms," Measurement Science and Technology, 20(7), 075501, 2009

[20] T. Latychevskaia and H.-W. Fink, "Practical algorithms for simulation and reconstruction of digital in-line holograms," Applied Optics, vol. 54, no. 9, p. 2424, 2015.

[21] F. C. A. Groen, I. T. Young, and G. Ligthart, "A comparison of different focus functions for use in autofocus algorithms," Cytometry, vol. 6, no. 2, pp. 81-91, 1985.

[22] Y. Zhang, H. Wang, Y. Wu, M. Tamamitsu, and A. Ozcan, "Edge sparsity criterion for robust holographic autofocusing," Optics Letters, vol. 42, no. 19, p. 3824, 2017.

[23] Local standard deviation of image - MATLAB stdfilt. (2019). [Online]. https://www.mathworks.com/help/images/ref/stdfilt.html

[24] T. H. et al Cormen, Introduction to algorithms. Cambridge, MA: MIT Press, 2009, pp. 414-450.

[25] K. Turkowski, "Filters For Common Resampling Tasks," Graphics Gems, pp. 147-165, 1990.

[26] M. Tiihonen, T. Jokela, L. Makkonen and G.-J. Bluemink, "VTT Icing Wind Tunnel 2.0," in Winterwind International Wind Energy $\begin{array}{lll}\text { Conference } 2016 . & \text { [Online]. Available: }\end{array}$ http://cris.vtt.fi/ws/files/23631741/VTT_Icing_Wind_Tunnel_2_0.pdf 